\title{
Índice de acessibilidade para comparação dos modos de transporte privado e coletivo
}

\author{
Accessibility index for comparison between private and \\ collective transport modes
}

Lilian dos Santos Fontes Pereira Bracarense ${ }^{[0]}$, Jéssica Oliveira Nunes Ferreira ${ }^{[b]}$

\author{
[a] Universidade Federal do Tocantins (UFT), Palmas, TO, Brasil \\ [b] Universidade de Brasília (UnB), Brasília, DF, Brasil
}

\section{Resumo}

Visando uma melhoria qualitativa e/ou quantitativa dos serviços de transporte ofertados e de sua infraestrutura base, observa-se a necessidade de oferecer instrumentos de planejamento que avaliem a acessibilidade, de forma a diagnosticar o nível de atendimento aos usuários pelos diferentes modos de transporte. 0 presente trabalho propôs a formulação de um índice que permita a comparação da acessibilidade dos modos de transporte privado e coletivo. A proposta é resultado de amplo estudo bibliográfico, buscando-se analisar características, limitações e aplicabilidade de índices desenvolvidos em estudos anteriores. A verificação da aplicabilidade da proposta foi feita com um estudo de caso na cidade de Palmas, TO. 0 índice formulado reúne variáveis que permitem sua aplicação tanto ao modo privado como ao coletivo, traduzindo em termos de tempo de viagem diferentes variáveis que caracterizam cada modo. Como resultado da análise é possível avaliar de forma integrada e espacializada a acessibilidade a transportes para diferentes categorias de usuários, além de permitir a simulação de intervenções, auxiliando no processo de planejamento urbano e de transportes.

Palavras-chave: Índice de acessibilidade. Planejamento urbano e de transportes. Transporte privado e coletivo.

\section{Abstract}

Aiming a qualitative and/or quantitative improvement of the offered transport services and its base infrastructure, it is observed the need to provide tools to evaluate accessibility, in order to diagnose the level of service to the users by the different modes of transport. This paper proposes to formulate an index allowing comparison of the accessibility of private and collective transport modes. The proposal is the result of extensive literature research, seeking to analyze characteristics, limitations and applicability of indices developed in previous studies. The proposal applicability was verified in a study developed in the city Palmas-TO. The index formulated gathers variables that allow its application to both private and collective modes, translating in terms of travel time different variables that characterize each mode. As a result of the analysis it is possible to evaluate in an integrated and spatialized way the accessibility to transport for different categories of users, besides allowing the simulation of interventions, aiding in the process of urban and transportation planning.

Keywords: Accessibility index. Urban and transport planning. Private and collective transport.

LSFPB é engenheira civil, professora da Universidade Federal do Tocantins (UFT), doutora em Transportes pela Universidade de Brasília (UnB), e-mail: lilianfontes@uft.edu.br

JONF é engenheira civil, mestre em Transportes pela Universidade de Brasília (UnB), e-mail: jessicaoliveir4@gmail.com 


\section{Introdução}

Atualmente, o termo acessibilidade é bastante usado no âmbito de planejamento urbano e de planejamento dos transportes. Em geral, os estudos presentes na literatura a respeito desse tema objetivam medir a facilidade de todos os grupos sociais acessarem destinos ou serviços de transportes. Gutierrez (2012) defende que $\mathrm{o}$ acesso a direitos e à igualdade de oportunidades remete a problemas de acessibilidade e também a ações programáticas orientadas para torná-lo efetivo. A acessibilidade é, portanto, o nexo principal entre o direito à mobilidade e o direito à cidade. É necessário, então, entender os múltiplos conceitos de acessibilidade e investigar como podem ser avaliados, de forma a contribuir para um planejamento integrado.

Diversos autores trazem a conceituação do termo acessibilidade - Ingram (1971), Morris et al. (1979), Jones (1981), Sales (1996), Raia (2000), Aguiar (2010). Em resumo, o conceito de acessibilidade que será tratado neste trabalho está relacionado ao acesso aos lugares (atividades ou destinos desejados), o qual depende do deslocamento dos indivíduos para este fim, seja por meio de modos motorizados e/ou não motorizados cuja escolha depende, por sua vez, das características físicas de cada indivíduo, das condições financeiras, as condições temporais (rapidez) e da distribuição espacial das atividades, a qual influencia na distância, tempo e/ou custo monetário dispendido no deslocamento. 0 nível de acessibilidade é menor à medida que se aumenta a distância-custo (custo de viagem em termos de tempo, distância ou custo monetário) de deslocamento entre dois pontos; assim, se as condições de mobilidade nesse percurso forem favoráveis, ou seja, o esforço de deslocamento for menos oneroso, aumenta-se a acessibilidade (Raia, 2000).

Entendendo a amplitude do tema, esse trabalho busca desenvolver uma ferramenta de avaliação da acessibilidade a sistemas de transportes que possa alimentar com informações consolidadas análises multidisciplinares que envolvam diversas dimensões da mobilidade urbana. Sob esse ponto de vista, observa-se a necessidade de oferecer instrumentos que avaliem a acessibilidade de forma a diagnosticar o nível de atendimento aos usuários pelos diferentes modos de transporte e a avaliar a necessidade de melhorias e investimentos em transporte em cidades de médio e grande porte.
Um dos aspectos considerados relevantes no desenvolvimento do trabalho foi a possibilidade de comparar modos de transporte motorizados, permitindo analisar como as políticas de transporte têm atendido cada um dos modos e se esse atendimento está alinhado com as diretrizes de melhoria dos serviços de transporte público e desestímulo ao uso do transporte individual.

Nesse sentido, o presente trabalho propõe a formulação de um índice que permita a comparação da acessibilidade dos modos de transporte individual por veículos particulares e transporte coletivo regular, em relação a uma mesma distância-custo, a qual representará a acessibilidade aos destinos-chave do centro da cidade a serem considerados.

Para permitir a comparação da acessibilidade dos diferentes modos de transporte, o índice precisa se basear nos mesmos critérios objetivos, sendo preservadas as especificidades de cada modo, e relevância desses critérios para o usuário. 0 índice proposto é resultado de amplo estudo bibliográfico, buscando-se analisar características, limitações e aplicabilidade aos modos motorizados de índices desenvolvidos por outros autores.

\section{Acessibilidade no âmbito de transportes}

O conceito de acessibilidade no âmbito dos transportes pode ser entendido a partir de dois focos: acessibilidade a destinos e acessibilidade ao sistema de transporte.

Essa divisão é sugerida e/ou subentendida em vários estudos pesquisados, a citar os trabalhos dos autores: Vickerman (1974), Jones (1981), Januário (1995), Pires (2000), Litman (2008 apud Guimarães Rodrigues, 2011), Oralu (2005 apud Dias, 2008) e Lemos (2008). Apesar da distinção da acessibilidade nesses dois conceitos, eles se complementam.

Em resumo, a acessibilidade a destinos é caracterizada como facilidade ou dificuldade de alcançar um local desejado (Vasconcellos, 2002; Ingram, 1971; VTPI, 2004 apud Melo, 2005; Brasil, 2006; Litman, 2008), oportunidade ou potencial de realização das viagens necessárias para atingir as atividades esperadas, sendo o tempo e a distância medidas de separação que limitam o número de oportunidades a serem alcançadas (Morris et al., 1979) e o conforto e 
conveniência para alcançar um determinado destino (Dalvi, 1978; Schoon et al., 1999).

É importante destacar a relação entre a acessibilidade a destinos e o uso e ocupação do solo. De acordo com Davidson (1995), acessibilidade influencia na forma como as atividades são organizadas numa área, ou seja, como elas se distribuem no espaço. Para Vasconcellos (2002), a acessibilidade a destinos é o indicador mais direto dos efeitos de um sistema de transporte específico. Segundo o autor, a acessibilidade é influenciada por vários fatores relacionados tanto aos serviços de transporte ofertado quanto aos elementos geográficos; como exemplos: as características espaciais da cidade, a localização das atividades e o nível de serviço do sistema de transporte.

A acessibilidade ao sistema de transporte mede a facilidade dos usuários de um sistema de transporte acessá-lo em sua região de moradia, trabalho e outros locais. São características que afetam a acessibilidade ao sistema de transportes: distância de caminhada da origem ao ponto de embarque (Ferraz \& Torres, 2004; Santos, 2005); proximidade do itinerário das atividades pretendidas pelo usuário (Batista \& Senne, 2000); infraestrutura e qualidade do sistema de transportes (Januário, 1995; Goto, 2000; Ferreira \& Sanches, 2001); localização dos pontos de embarque e frequência das linhas (EBTU, 1998); aspectos físicos e econômicos (Costa, 2008). Apesar do conceito de acessibilidade ser amplo no âmbito dos transportes, as múltiplas definições convergem para um sentido comum interpretado como a facilidade, potencial ou oportunidade de acessar as diferentes atividades distribuídas em uma área, por meio de um sistema de transporte individual ou coletivo, cujo esforço de superação depende: da localização dessas atividades no espaço; da qualidade, quantidade ou infraestrutura oferecida pelo sistema escolhido; das características espaciais da rede viária e, no caso do transporte público, do posicionamento dos pontos de parada.

Por uma perspectiva objetiva, a partir da conceituação dada pelos diferentes autores, observa-se a importância das distâncias de caminhada dos usuários do início da viagem até o ponto de embarque e do local de desembarque até o destino final. Nesse sentido, a localização dos pontos de parada influencia diretamente o maior ou menor acesso ao sistema de ônibus, reflexo do esforço necessário para o deslocamento, isto é, a distância de caminhada. 0 uso e a ocupação do solo, as condições de tráfego, dentre outros aspectos, podem caracterizar a flexibilidade de distribuição de pontos de embarque/desembarque. Cabe ressaltar também os aspectos subjetivos relacionados ao conceito de acessibilidade, visto que a percepção em relação à adequação desses parâmetros pode variar entre diferentes grupos de usuários, por interferência de suas limitações físicas e sociais.

\section{Indicadores de acessibilidade}

Para Gudmundsson (2004 apud Costa, 2008) os indicadores são variáveis que podem funcionar como "balizadores" na elaboração de políticas e em análises técnicas, bem como ajudar a operar objetivos e tornar determinados sistemas gerenciáveis de forma menos complexa, além disso, eles revelam as condições dos sistemas, políticas ou organizações por meio de medidas de desempenho referentes às metas ou objetivos analisados.

Um índice, por sua vez, é criado a partir da combinação de indicadores e representa uma medida única, a qual compõe uma maior quantidade de informações (Saisana et al., 2005). Os índices desse tipo são atraentes, pois resumem as informações relacionadas às questões complexas, porém quando não se apoiam nos indicadores originais podem implicar resultados simplistas.

Raia (2000) chegou a uma classificação geral dos indicadores de acessibilidade a partir de estudos anteriores de diversos autores, classificando os indicadores de acessibilidade em:

i) Indicadores do tipo atributos de rede:

a. Conectividade de nó nas interligações do sistema de transporte: Indica se dois pontos estão conectados fisicamente por um sistema de transporte. Nesse sentido, a acessibilidade a destinos de uma zona é medida pelo número de zonas que estão conectadas a essa zona pelo sistema de transporte;

b. Acessibilidade temporal: Considera os períodos em que não há disponibilidade do sistema de transporte, ou seja, as horas e/ou dias em que certas linhas do transporte coletivo não funcionam;

c. Separação espacial: Acessibilidade a destinos é medida utilizando-se indicadores de separação espacial, tais como a distância entre zonas e/ou 
o tempo médio da viagem entre zonas ou outro mais complexo.

ii) Indicadores do tipo quantidade de viagens: Consideram o custo da viagem e a probabilidade das viagens ocorrerem;

iii) Indicadores do tipo oferta do sistema de transporte: Utilizam como parâmetros o número de linhas que servem a zona de estudo, a frequência do sistema e a área dessa zona;

iv) Indicadores que usam dados agregados que combinam aspectos de transporte e uso do solo;

v) Indicadores que usam dados desagregados que combinam aspectos de transporte e uso do solo.
Para a melhor compreensão a respeito dos indicadores de acessibilidade, o presente trabalho adotará a classificação geral dos indicadores proposta por Raia (2000), relacionando-a aos conceitos de acessibilidade vistos anteriormente. Os principais indicadores encontrados na literatura estão listados na Tabela 1.

De acordo com Vickerman (1974), é difícil quantificar ou mensurar a acessibilidade de forma precisa por ela englobar tanto os elementos distribuídos geograficamente no espaço quanto as características dos sistemas de transporte. Além disso, em seu sentido mais amplo, o conceito de acessibilidade deve considerar a percepção dos diferentes grupos de usuários e suas restrições, aumentando sua complexidade de mensuração. As dificuldades quanto à coleta de dados

Tabela 1 - Indícies de acessibilidade de estudos anteriores

\begin{tabular}{|c|c|c|c|}
\hline Tipo & Autor & Formulação & Variáveis \\
\hline \multirow{3}{*}{$\begin{array}{l}\text { Indicadores de } \\
\text { separação espacial }\end{array}$} & Ingram (1971) & $A_{i}=\sum_{j}^{n} a_{i j}$ & $\begin{array}{l}\text { A: Acessibilidade integral do ponto } i_{i} \\
a_{i} \text { :Acessibilidade relativa entre os pontos ie } i \text {; } \\
\text { : Número total de pontos da rede }\end{array}$ \\
\hline & Allen et al. (1993) & $A_{i}=\frac{1}{N-1} \times \sum_{j}^{N} C_{i j}$ & $\begin{array}{l}\text { A:Acessibilidade da zona i } i_{i} \\
\text { N: Número de pontos utilizados no cálculo; } \\
C_{i}: \text { Custo despendido para se deslocar entre as zonas ie } i\end{array}$ \\
\hline & Davidson (1995) & $A_{i}=\sum_{j}^{n} S_{j} \times f\left(C_{i j}\right)$ &  \\
\hline Quantidade de viagens & Dodgson (1974) & $\begin{array}{c}A_{i}=\sum_{j} P_{i j} \cdot C_{i j}^{\infty} \\
P_{i j}=\frac{W_{j}}{C_{i j}} / \sum_{J} \frac{W_{j}}{C_{i j}}\end{array}$ & 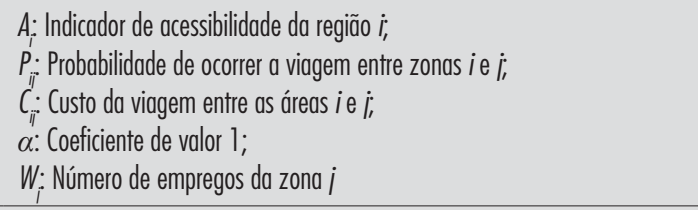 \\
\hline \multirow{3}{*}{$\begin{array}{l}\text { Oferta do sistema de } \\
\text { transportes }\end{array}$} & Bruton (1979) & $A_{i}=\frac{\sum_{i} \sqrt{F_{m, i}^{Z}}}{\sqrt{S_{i}}}$ & 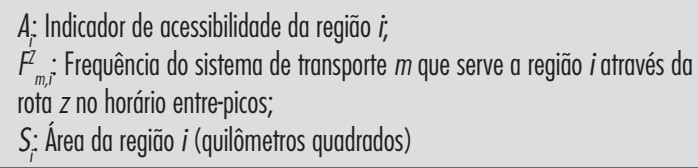 \\
\hline & $\begin{array}{l}\text { Januário } \\
\text { (1995) }\end{array}$ & $A_{i}=\sum_{j}^{n} L_{i j} f\left(c_{i j}\right)$ & 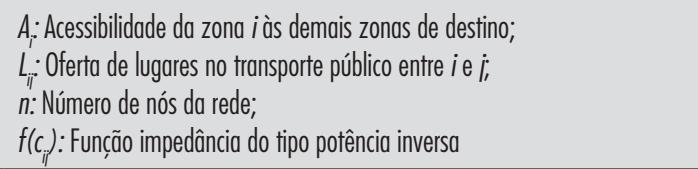 \\
\hline & Henk \& Hubbard (1996) & $I T S A=\frac{I_{c o b}+I_{f}+I_{c a p}}{3}$ & 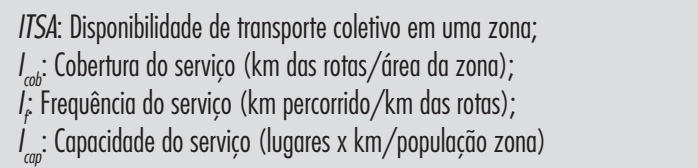 \\
\hline
\end{tabular}

Fonte: Bracarense \& Ferreira (2014). 
Tabela 1 - Continuação...

\begin{tabular}{|c|c|c|c|}
\hline Tipo & Autor & Formulação & Variáveis \\
\hline $\begin{array}{l}\text { Oferta do sistema de } \\
\text { transportes }\end{array}$ & Dias (2008) & $\begin{array}{c}X_{i, p}=\frac{X_{i}-\min \left(X_{i}\right)}{\max \left(X_{i}\right)-\min \left(X_{i}\right)} \\
X_{i, \text { novo }}=1 \cdot X_{i, p} \\
I A_{\mathbb{P}}=\sum_{i=n}^{N} X_{I} \cdot W_{P}\end{array}$ & 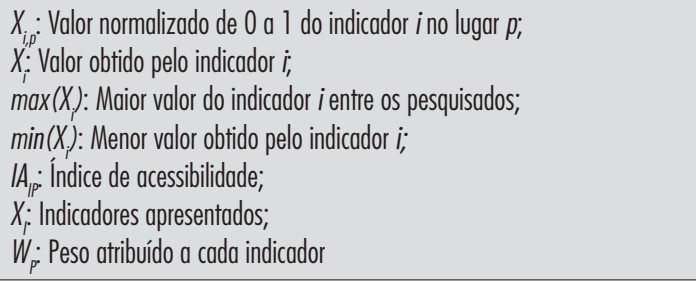 \\
\hline \multirow{3}{*}{$\begin{array}{l}\text { Indicadores que usam } \\
\text { dados agregados que } \\
\text { combinam aspectos } \\
\text { de transporte e uso } \\
\text { do solo }\end{array}$} & $\begin{array}{l}\text { Vickerman (1974), Dalvi } \\
\text { 1978), Koenig (1980), } \\
\text { Hanson (1995) }\end{array}$ & $A_{i}=\sum_{j} W_{j} . f\left(C_{i j}\right)$ & $\begin{array}{l}W_{i} \text { Número de oportunidades na zona } j \text { para dado motivo; } \\
f\left(C_{i)} \text { como } 1 / C^{a} \text { ii (Hanson, 1995), onde } C_{i j} \text { é distância ou tempo de viagem }\right. \\
\text { entre as zonas ie } j \text { e } \alpha \text { coeficiente que geralmente recebe valor } 1 \text { (um) }\end{array}$ \\
\hline & Deka (2002) & $\mathrm{DTC}_{i}=\frac{\sum_{r=1}^{m} C_{r} \times F_{r}}{A_{i}}$ & $\begin{array}{l}\text { DTC: Disponibilidade de transporte coletivo no setor } i_{i} \\
C_{i} \text { Q Quilometragem da linha } r \text { na área estendida do setor } i_{;} \\
F_{i} \text { Frequência do serviço na rota r, no pico da manhãa; } \\
\text { m: Número de linhas que atravessam o setor estendido; } \\
A_{i} \text { : Área do setor estendido }\end{array}$ \\
\hline & $\begin{array}{l}\text { Raia et al. } \\
\text { (1997) }\end{array}$ & $A_{i}=\sum_{j}$ Dens $_{j} \cdot\left(\text { Dist }_{i j}\right)^{-\alpha}$ & 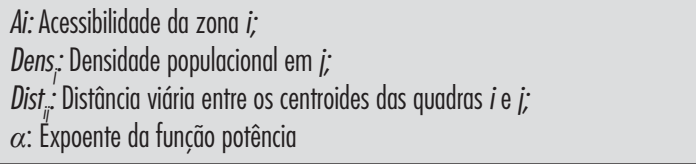 \\
\hline \multirow{3}{*}{$\begin{array}{l}\text { Indicadores que usam } \\
\text { dados desagregados } \\
\text { que combinam } \\
\text { aspectos de transporte } \\
\text { e uso do solo }\end{array}$} & Formulação geral & $\begin{array}{l}A_{i}=\sum_{j} W_{j} \cdot f\left(c_{i j}\right) \\
f\left(c_{i i}\right)=1 \text { se } c_{i i} \leq C \\
f\left(c_{i i}\right)=0 \text { se } c_{i j} \geq C\end{array}$ & $\begin{array}{l}\text { C: Medida de contorno (referência); } \\
W_{i} \text { N Número de oportunidades na zona; } \\
c_{i i} \text { C Custo despendido de viagem entre as zonas ie } i\end{array}$ \\
\hline & Tagore \& Sikdar（1995) & $A_{i}=\left(\sum_{j}^{n} S_{j} \times f\left(t_{i j}\right) \times e^{\left(\tilde{\mathrm{a}} \times M_{i}\right)}\right) / \sum$ & 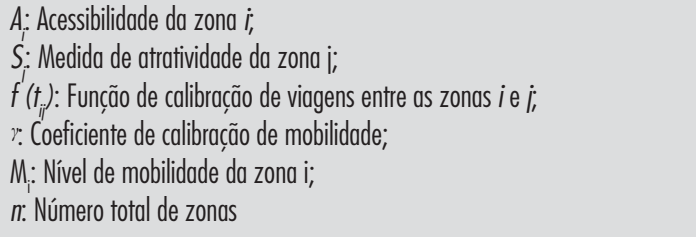 \\
\hline & Wang (2003) & $A_{i}^{G}=\sum_{j=1}^{J}\left(\frac{O_{j}^{g} \times f\left(t_{i j}\right)}{\sum_{k=1}^{K} W_{k}^{g} \times f\left(t_{k i}\right)}\right)$ & 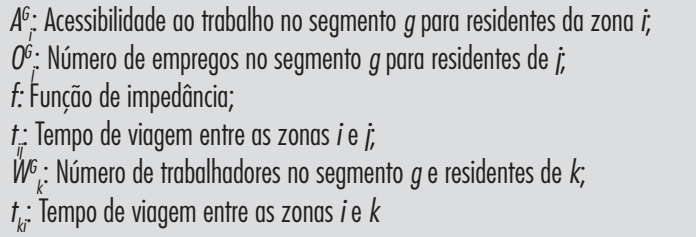 \\
\hline
\end{tabular}

Fonte: Bracarense \& Ferreira (2014).

e/ou aplicação de determinado indicador levará à adaptação do mesmo ou a sua eliminação na análise de um sistema ou fenômeno em questão, assim novos indicadores poderão ser considerados.

A identificação de indicadores pode ser feita através de pesquisa de experiências anteriores sobre o sistema ou fenômeno que se deseja medir, sendo fundamental o envolvimento de especialistas nesse processo. A escolha dos indicadores tem como critérios gerais a validade científica, a relevância, o custo, a atratividade, a representatividade e outros. Assim, os indicadores e seus critérios devem ser comparados para identificar a ausência ou presença de propriedades que possibilitem optar por um ou outro indicador e/ou selecionar quais indicadores irão compor um conjunto (Costa, 2008).

Dentre os indicadores pesquisados, observa-se que muitos deles requerem variáveis de difícil mensuração, ou sem o nível de detalhamento adequado para seu cálculo, tais como acessibilidade relativa 
(Ingram, 1971) e atratividade da zona (Davidson, 1995; Tagore \& Sikdar, 1995). Outros indicadores são específicos de um único modo de transporte (Bruton, 1979; Januário, 1995; Henk \& Hubbard, 1996; Dias, 2008), o que impossibilita uma análise comparativa entre modos de transporte distintos. A análise desses indicadores permitiu identificar a necessidade de um único indicador que possa ser aplicado em uma análise comparativa entre diferentes modos e assim contribuir para diagnósticos e prognósticos que considerem o sistema de transportes como um todo em estudos de mobilidade urbana.

\section{Metodologia}

O desenvolvimento do estudo proposto se deu em duas etapas. Inicialmente foi desenvolvida a formulação teórica do índice de acessibilidade, a partir da revisão da literatura, considerando um grupo de usuários sem restrição de locomoção; em seguida, o índice proposto foi aplicado ao município de Palmas, empregando-se ferramentas de Sistema de Informação Geográfica.

\section{Etapa 1: Proposição do índice}

Com intuito de desenvolver um índice de acessibilidade que permita realizar análises comparativas entre modos de transporte, buscou-se relacionar as características e os tipos de indicadores mais adequados, considerando-se as variáveis fundamentais para caracterizar cada modo, sem excluir nenhum modo:

- Transporte individual

a) Abordagem: Acessibilidade aos destinos;

b) Indicadores: Indicadores do tipo atributos de rede.

Devem ser considerados indicadores que refletem ou influenciam o custo da viagem medido pelo tempo, distância ou custo. Tais indicadores de separação espacial estão relacionados à configuração da rede viária, por exemplo: capacidade das vias (número de faixas de tráfego); tipo e estado de conservação do pavimento; velocidade operacional (permitida) da rede viária; presença de intersecções com/sem sinalização semafórica e/ou canalização de tráfego por rotatórias ou rótulas etc.

\section{- Transporte coletivo}

a) Abordagem: Acessibilidade aos destinos e acessibilidade ao sistema de transporte; b) Indicadores: Indicadores do tipo atributos de rede e de oferta do sistema de transportes.

- Acessibilidade locacional: Referente à localização dos pontos de parada próximos a origem e destino das viagens. Assim, a acessibilidade locacional é medida pela distância ou tempo despendido pelo usuário até o ponto de embarque e/ou do ponto de desembarque até o destino pretendido;

- Acessibilidade temporal: Indicada pela frequência das linhas de ônibus que atendem os pontos de embarque/desembarque.

Apenas serão considerados indicadores que caracterizam a acessibilidade locacional e temporal ao modo de transporte coletivo, portanto serão descartados parâmetros socioeconômicos como a renda familiar e a tarifa/custo do transporte e, também, de conforto nos veículos e nos pontos de parada, os quais fogem da proposta deste trabalho (medir o acesso físico ao espaço urbano e ao sistema de transporte). A título de exemplo de variáveis que compõem esses indicadores têm-se: o número de linhas que servem a zona de estudo; a frequência do sistema; distribuição e localização dos pontos de parada; o itinerário das linhas; tempo ou distância de caminhada e tempo de espera nos pontos de parada para ter acesso ao veículo; e a velocidade média operacional do veículo de transporte coletivo.

Definidas as características gerais que o índice deve atender, foram selecionadas as variáveis mais representativas desses indicadores, de modo a minimizar o número de variáveis envolvidas. As variáveis selecionadas foram: tempo de viagem, tempo de percurso motorizado, tempo de percurso a pé, frequência de viagem, pontos de destino-chave.

\section{Etapa 2: Estudo de caso}

A área estudada para aplicação do modelo e obtenção do índice de acessibilidade dos transportes motorizados individual e coletivo foi a região central de Palmas, TO.

A cidade de Palmas é considerada a última capital brasileira planejada e teve sua pedra fundamental de construção lançada em 20 de maio de 1989. Em relação à infraestrutura do sistema viário, os principais eixos de transporte - Avenida Teotônio Segurado, Via Auxiliar e Avenida JK - são áreas dotadas de infraestrutura 
de base; característica essa que a Lei Complementar n. 155/2007 do Plano Diretor Participativo de Palmas (PDPP) usou como referência para a determinação das Áreas de Ocupação Prioritária, sendo aquelas inseridas próximas aos eixos de deslocamento, como mostra a Figura 1.

Essa lei observou a localização de áreas produtivas integradas aos eixos de transporte e, então, priorizou a ocupação das quadras com infraestrutura, onde se estrutura o eixo de transporte, objetivando melhorar a acessibilidade da população e, consequentemente, o acesso aos bens e serviços urbanos. Sendo assim, as centralidades localizam-se ao longo das áreas de ocupação prioritária nas regiões norte e centro do município. Na região sul, a ocupação se deu de forma alheia ao previsto no plano inicial, apresentando uma configuração urbana diferente do restante da cidade.

No presente trabalho, a acessibilidade foi avaliada em relação ao espaço urbano norte e central de Palmas, $\mathrm{TO}$, sendo considerados o tempo gasto para se atingir um conjunto de destinos-chave, isto é, alguns pontos localizados na área central da capital (centroides), a partir de outros pontos de origem (nós da rede). Foi considerada a utilização de modo de transporte individual por veículos particulares e transporte coletivo regular, permitindo assim a obtenção e comparação dos índices de acessibilidade desses transportes motorizados. No total foram considerados 52 pontos de destino.

Para comparação dos índices de acessibilidade e verificação do método proposto, foram avaliados três cenários: transporte individual, transporte coletivo excluindo-se o parâmetro de frequência e transporte coletivo por meio do índice proposto.

Os dados de frequência e velocidade operacional das linhas de ônibus foram obtidos da planilha da Ordem de Serviço n. 6/2014, fornecida pela Secretaria Municipal de Acessibilidade, Mobilidade e Transporte. Quanto ao transporte por automóvel individual, seu indicador de acessibilidade demandou, também, uma base georreferenciada da geometria da rede viária e a sua velocidade operacional. A base de dados georreferenciada da rede viária foi a mesma utilizada para o transporte coletivo, a qual foi adquirida no SIGPALMAS. Já a velocidade média operacional foi obtida pela aplicação do Método do Veículo-Teste com Cronômetro, do Manual de Estudos de Tráfego do DNIT - Departamento Nacional de Infraestrutura de Transportes (DNIT, 2006). A Figura 2 apresenta os resultados obtidos representados em mapa.

Os mapas resultaram da avaliação multicritério integrada em um Sistema de Informação Geográfica (SIG), obtidos para cada modo de transporte (individual e coletivo), os quais apresentam os níveis de acessibilidade

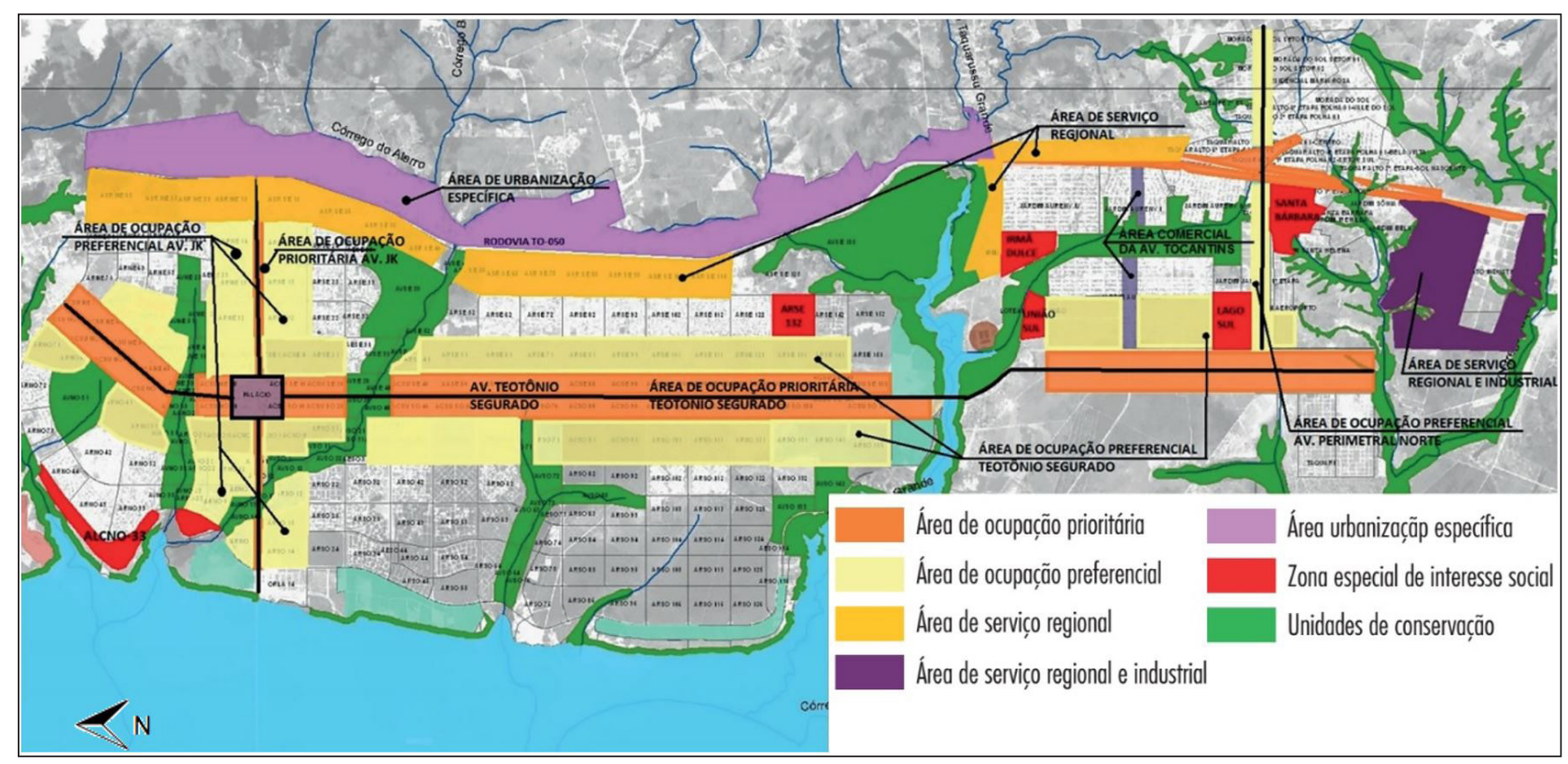

Figura 1 - Ordenamento Territorial de Palmas aprovado pela lei n. 155/2007 (PDPP)

Fonte: Palmas, 2007 apud Coriolano et al. (2013, p. 140).

urbe. Revista Brasileira de Gestão Urbana (Brazilian Journal of Urban Management), 2018 set./dez., 10(3), 600-613 
divididos em cinco classes normalizadas no intervalo entre 0 (zero) e 52 . 0 índice de acessibilidade foi calculado para cada nó da rede, em seguida construiu-se um modelo digital de terreno a partir dessa malha de nós, classificando-se os pontos em faixas de acordo com o valor do índice de acessibilidade. Considerou-se que, para a faixa entre 0,00 e 10,00 o nível de acessibilidade é ineficiente, entre 10,00 e 20,00 é de baixa eficiência, entre 20,00 e 30,00 é de média eficiência, entre 30,00 e 40,00, de boa eficiência e entre 40,00 e 52,00, de alta eficiência. Essa análise permite verificar quais áreas têm melhores ou piores condições de acessibilidade para os usuários dos modos de transporte individual e coletivo, sugerindo a necessidade de algumas intervenções de melhoria no desempenho do espaço.

\section{Resultados}

0 índice proposto para comparação da acessibilidade pelos modos de transporte coletivo e privado se baseia nas características físicas das redes de oferta existentes. Sendo assim, o índice proposto é dado pelas Equações 1 e 2:

$$
\begin{aligned}
& A_{i j}=\sum_{j}^{n}\left(1-\frac{T_{i j}-T_{\min }}{T_{\max }-T_{\min }}\right) \\
& T_{i j}=t_{i j}^{M}+t_{i j}^{N M}+\frac{60}{f_{i j}}
\end{aligned}
$$

em que:

$A_{i j}$ : é o índice de acessibilidade do nó $i$ para os destinos $j$; $T_{i j}$ : é o tempo de viagem do nó $i$ para o destino $j$ em minutos;

$T_{\max }$ : é o máximo tempo de viagem de $i$ até $j$ para o qual a viagem pode ser considerada viável para o usuário, em minutos;

$T_{\text {min }}$ : é o menor tempo de viagem de $i$ até $j$ encontrado para a rede de transportes analisada, em minutos;

$f_{i j}$ : é a frequência de viagem do veículo que atende a rota do nó $i$ para o destino $j$ em veículos/hora; $t_{i j}^{M}$ : é o tempo de percurso entre o nó $i$ e o destino $j$ pelo modo motorizado, em minutos;

$t_{i j}^{N M}$ : é o tempo de percurso a pé, anterior ao embarque ou após o desembarque, entre o nó $i$ e o destino $j$, em minutos;

$n$ : é o número de destinos-chave do tipo $j$ para o qual se analisa a acessibilidade do nó $i$.
A variável tempo de viagem é decomposta em tempo de espera e tempo de percurso no transporte coletivo. O tempo de espera foi introduzido na formulação a partir da frequência do veículo. Para o modo privado não há tempo de espera para o usuário, sendo desprezada a parcela que inclui a frequência. Para o modo coletivo deve ser considerada a maior frequência horária das linhas que atendem o percurso de i para j, ou a soma das frequências dessas linhas, quando os horários não forem coincidentes.

As demais variáveis mencionadas anteriormente influenciam no tempo de percurso, que é obtido como resultado de uma análise física e operacional da oferta. Sendo assim, as variáveis que permitem a obtenção dos tempos de percurso são:

- Velocidade operacional: Variável fundamental para o cálculo dos tempos de percurso. É obtida a partir de pesquisa de velocidade e retardamento realizada nas vias da área de estudo. Por sua vez, a velocidade é influenciada pelo estado de conservação do pavimento, tipo de controle de interseções, capacidade viária e deve ser determinada para cada modo de transporte analisado. Na impossibilidade de se realizar a pesquisa de velocidade em todas as vias da área de estudo, pode ser feita uma pesquisa amostral para cada grupo de vias com características geométricas e de fluxo semelhantes. Outra alternativa é a utilização dos resultados de uma alocação de tráfego, devidamente calibrada, para a região de estudo;

- Distribuição e localização dos pontos de parada: A oferta espacial dos pontos de parada define a distância que deve ser percorrida a partir de cada origem $i$ até se acessar a linha de transporte e a partir desse acesso até o destino $j$. Essa distância é percorrida, na maioria das vezes, a pé pelo usuário de transporte coletivo, devendo ser considerada a velocidade de caminhada no cálculo do tempo de percurso;

- Itinerário das linhas: Semelhanteà localização dos pontos de parada, o itinerário definirá a distância de cada origem $i$ ao sistema de transportes e do sistema de transportes a cada destino $j$.

Como resultado, para cada origem $i$ é obtido um número absoluto que representa a acessibilidade desse ponto ao conjunto de destinos considerados. 
A escala dos resultados será variável em função do número $n$ de destinos $j$ que compõem o somatório. O índice proposto pode ser calculado para um único par origem/destino ou para toda a rede, de todos os nós para os destinos selecionados, com auxílio de ferramentas de geoprocessamento. Permite obter, assim, uma representação espacial identificando áreas com maior ou menor acessibilidade para cada modo de transporte.

Aplicando-se o índice proposto ao município de Palmas, TO, foram obtidos os mapas representados na Figura 2. Comparando-se os dois mapas referentes ao transporte coletivo pode-se verificar que para os mesmos intervalos de valores dos índices calculados, a

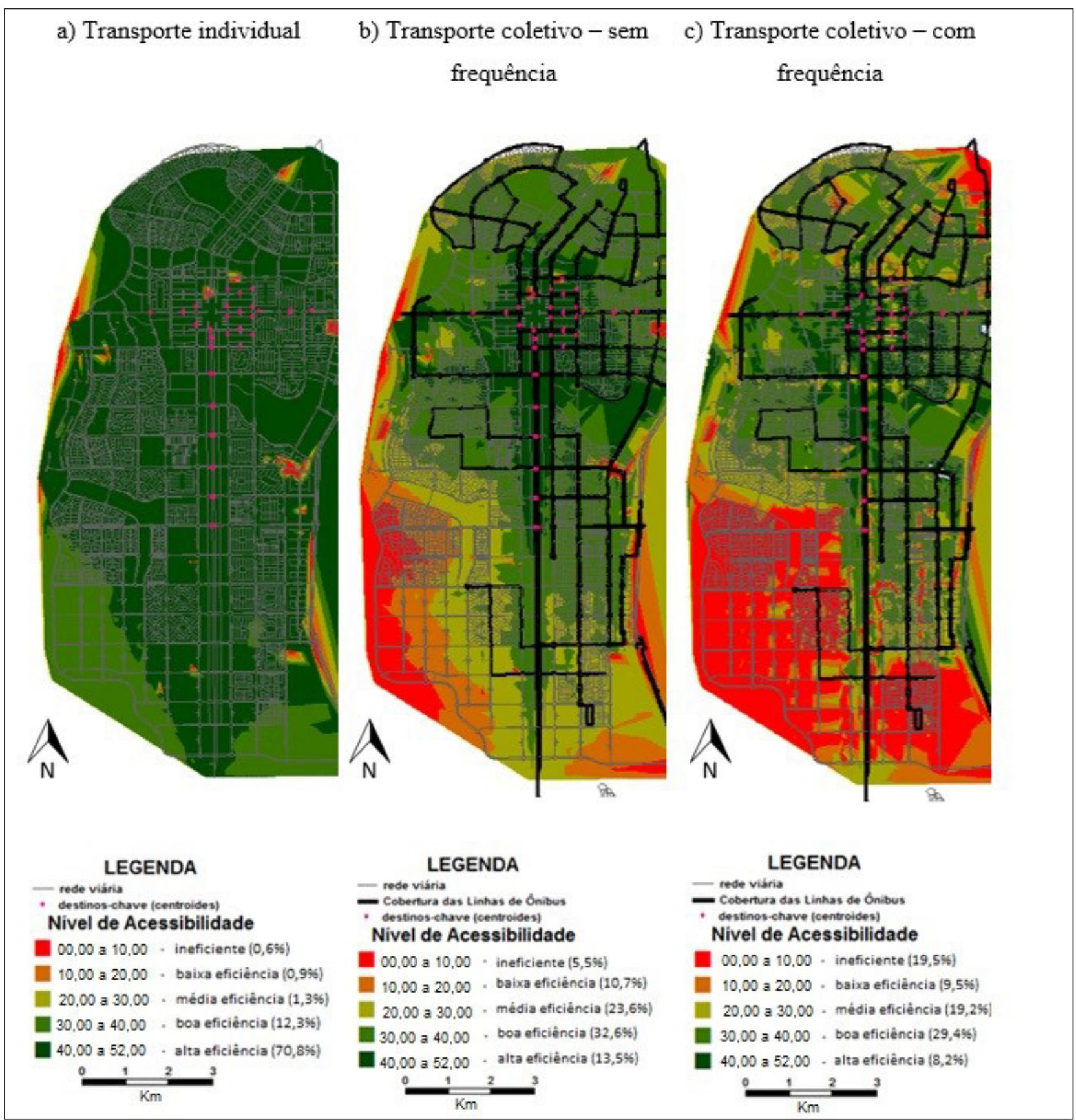

Figura 2 - Mapas de acessibilidade por modo de transporte Fonte: elaboração própria. 
porcentagem que representa o nível de acessibilidade péssimo é consideravelmente maior no mapa em que foi atribuído o critério físico da frequência no cálculo do tempo de viagem, o mapa da Figura 2c, conforme mostram os "bolsões" na cor vermelho que aparecem em boa parte da porção sudoeste e no extremo nordeste da área de estudo, à medida que as zonas consideradas com boa e ótima acessibilidade, respectivamente nas cores em verde claro e escuro, são reduzidas.

0 mapa da Figura 2c representa melhor a realidade dos usuários do sistema de transporte coletivo na região central de Palmas, pois contabiliza o tempo de espera do indivíduo para ter acesso ao embarque, sendo que usuários que necessitam aguardar longos períodos pelo embarque têm mais dificuldade de acesso do que usuários de uma localidade atendida por uma linha com tempo de espera menor. No caso da porção sudoeste do mapa, apesar da existência da linha de transporte, a região pode ter uma acessibilidade baixa devido à baixa frequência de operação do serviço existente, mostrando a relevância de se considerar tal parâmetro operacional no cálculo do índice.

Para efeito comparativo entre o modo privado e coletivo serão comparados os mapas das Figuras 2a e 2c. A Figura 3 representa graficamente os resultados encontrados.

Com relação ao índice de acessibilidade do transporte individual, representado na Figura 2a, observa-se que a maior parte da área de estudo apresenta um nível de acessibilidade de alta eficiência (cerca de 70,8\%), como mostra o enorme "bolsão" na cor verde-escuro. A faixa de valores dos índices de 30,00 a 40,00, a qual classifica o nível de acessibilidade como de boa eficiência, também representa uma porcentagem considerável dentro da área de estudo, cerca de 12,3\%, na zona situada ao extremo sul.

No caso dos índices do transporte coletivo, representados no mapa da Figura 2c, o número de nós da rede classificados na faixa de valores de 40,00 a 52,00, na qual o nível de acessibilidade é considerado de alta eficiência, chega a ser 8,634 vezes menor; e o seu "bolsão" (em verde-escuro) circunda quase a totalidade dos destinos-chave. Os "bolsões" da porção sudeste, especialmente o localizado no entorno da rodovia, ganharam proporções maiores, que abrangeram o extremo sul da área avaliada, seguindo até pouco mais da metade da área sudoeste da cidade; de forma que, juntas, as três faixas de intervalo com índices de 0,00 a 30,00 representam pouco mais de $48 \%$ da área de estudo; da mesma forma o nível de acessibilidade classificado como boa eficiência que, para o transporte coletivo, representou $29,4 \%$ da área de avaliação.

A diferença entre os valores máximos dos índices encontrados para os dois modos de transporte é pequena $-49,35$ do transporte individual e 47,66 do coletivo, visto que foram consideradas para o cálculo dos tempos reais de percurso de cada modo as respectivas velocidades operacionais no período

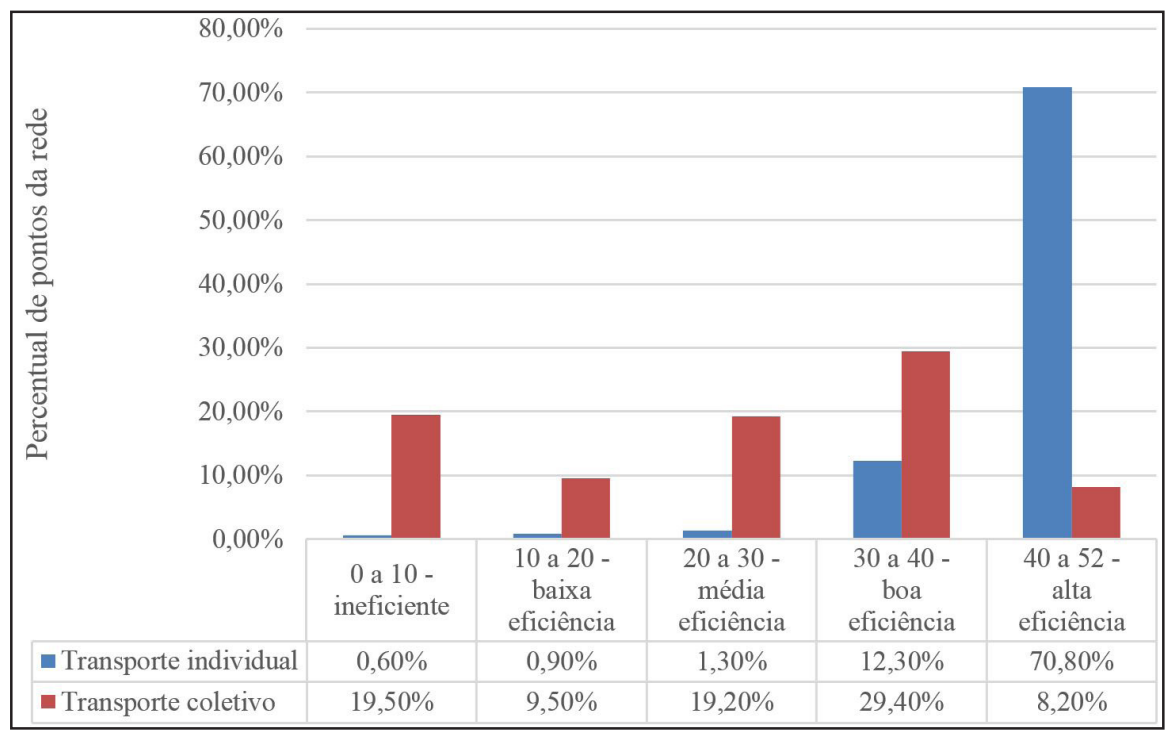

Figura 3 - Classificacãa dos pontos da rede quanto à eficiência do nível de acessibilidade para os modos de transporte individual e coletivo Fonte: elaboracão própria. 
de pico (de 3 horas), entre a manhã e a tarde, período em que os dois sistemas operam com velocidades semelhantes devido às impedâncias encontradas nos trechos a percorrer até os destinos-chave, por exemplo, os congestionamentos nas rotatórias e interseções semaforizadas. Como as velocidades de percurso atingem valores semelhantes, a diferença entre os valores máximos observados se deve aos tempos de espera presentes no modo coletivo, que acabam por reduzir a velocidade média de viagem desse modo.

A comparação evidencia a necessidade de melhorias nos serviços e no atendimento aos usuários do transporte coletivo, permitindo identificar as áreas mais carentes dessas melhorias. A análise de acessibilidade contribui para avaliar a situação do município em relação às diretrizes de mobilidade urbana sustentável. No Brasil, o direito à mobilidade urbana começou a ser institucionalizado a partir do Estatuto da Cidade, de 2001, e evoluiu até a promulgação da lei n. 12.857/2012. A Lei da Mobilidade Urbana determina a integração dos diferentes modos de transporte e a melhoria da acessibilidade dos indivíduos e cargas nos municípios, objetivo da PNMU, por meio do planejamento e da gestão dos sistemas de mobilidade urbana pelos órgãos competentes incumbidos de "planejar e coordenar os diferentes modos e serviços" e "[...] avaliar e fiscalizar os serviços e monitorar desempenhos, garantindo a consecução das metas de universalização e de qualidade [...]" (Brasil, 2012, art. 22). Para que tais políticas sejam implementadas nos municípios, é necessário o fomento de mais pesquisas sobre o assunto e de preferência que estudos se realizem nas próprias cidades, para que os problemas sejam representados mais próximos da realidade local. Nesse sentido, o índice de acessibilidade proposto permite que cada cidade avalie a eficiência de seu sistema de transporte e possa priorizar os investimentos de forma mais equilibrada, para suprir as deficiências detectadas.

Ao considerar os destinos-chave como elemento central do qual se deseja avaliar a acessibilidade, o índice permite aplicar a visão do planejamento de transporte integrado ao planejamento urbano, em conformidade com a óptica da mobilidade urbana sustentável. Sobre esse aspecto, Banister (2008) identifica quatro pilares da mobilidade urbana sustentável, que se refletem na melhoria da acessibilidade: i) Substituição, com a redução da necessidade de realização de viagens a partir da crescente realização de atividades de forma remota; ii) Incentivo à troca modal, com políticas de transporte que estimulem a redução do uso do automóvel e estimulem uso de modos coletivos e não motorizados; iii) Redução do comprimento de viagens, com mudanças no uso do solo, promovendo diversidade e complementaridade dos usos; e iv) Melhoria progressiva da eficiência do sistema de transportes, com inovação tecnológica. Trata-se de uma lógica de planejamento voltada para gestão da demanda, e não para seu imediato atendimento, como tradicionalmente abordado.

Por essa abordagem, os problemas de transporte não podem ser examinados dissociados da cidade, devendo envolver questões urbanísticas, sociais, econômicas e políticas. Para ter sucesso, como conclui Hull (2008), o paradigma do transporte sustentável deve ser entendido por todos os setores, de forma que as políticas públicas convirjam para os mesmos objetivos no que tange a mobilidade urbana.

Em relação aos princípios da mobilidade urbana sustentável, observa-se que a infraestrutura disponível na cidade de Palmas ainda privilegia o automóvel, havendo necessidade de novas políticas para reverter esse quadro e aproximar a cidade das metas de sustentabilidade e das diretrizes da Lei da Mobilidade Urbana. Cabe ressaltar a importância de se analisar, juntamente com esses dados de acessibilidade, informações socioeconômicas, como densidade populacional e renda, a fim de se avaliar o volume e perfil da população impactada e propor soluções mais adequadas à realidade local.

\section{Considerações finais}

A proposta aplica o conceito de acessibilidade a destinos variáveis de indicadores de acessibilidade ao sistema de transportes, como a frequência. 0 índice proposto reúne variáveis que permitem sua aplicação tanto ao modo privado como ao coletivo, traduzindo em termos de tempo de viagem diferentes variáveis que caracterizam cada modo.

A formulação do índice produz um resultado normalizado, semelhante ao estudo de Dias (2008). 0 custo, utilizado por diversos autores como descrito na Tabela 1, é dado pelo tempo de viagem, decomposto em tempo de percurso e tempo de espera. Dessa forma, o índice pode ser aplicado a vários modos de transporte, permitindo uma comparação na mesma escala. 
0 índice é coerente com o conceito de acessibilidade, ou seja, quanto maior a facilidade de transporte maior é a acessibilidade. Observa-se que um maior valor do tempo de percurso tende a diminuir o valor do índice, enquanto a maior frequência tende a aumentá-lo, pois reduz o tempo de espera e, consequentemente, o tempo de viagem.

O índice proposto constitui uma ferramenta capaz de contribuir para o aprofundamento das análises de diagnóstico dos sistemas de transporte atuais e análise de cenários para avaliação de alternativas, principalmente pela possibilidade de aplicação espacial, considerando todos os nós de determinada rede.

0 modelo de avaliação da acessibilidade adotado nesta pesquisa para a obtenção do índice de acessibilidade resulta da combinação da análise multicritério integrada ao ambiente SIG (Sistemas de Informação Geográfica). Esse método multicritério permite que grandes números de dados e objetivos sejam avaliados conjuntamente, enquanto o uso de SIGs oferece mecanismos para combinar as várias informações e manipular os dados neles armazenados, constituindo potente ferramenta de processamento e análise espacial.

A aplicação do modelo para o caso de Palmas foi fundamental para a avaliação dos níveis de acessibilidade relativos aos destinos-chave, aí considerados quanto às condições dos usuários utentes (sem restrição de locomoção) desses dois modos de transporte. À medida que mais critérios são atribuídos na avaliação da acessibilidade, mais próximo da realidade são os resultados obtidos e, portanto, relevante a adoção do método multicritério de avaliação. Enquanto ferramenta de tomada de decisão, o modelo proposto contribui para que as formas de avaliação de alternativas sejam mais abrangentes, possibilitando que aspectos operacionais, no caso a frequência, sejam incorporados na análise.

Este trabalho contribui para o conhecimento e formulação de novas pesquisas sobre o tema, dada a fundamentação teórica que relaciona os conceitos de acessibilidade e mobilidade e a tipologia dos indicadores de acessibilidade envolvidos e, também, serve de apoio à tomada de decisão dos planejadores e administradores urbanos, a partir dos resultados apresentados e analisados nesta pesquisa, os quais relacionam os níveis de acessibilidade do espaço urbano de Palmas às condições de mobilidade dos usuários utentes dos transportes motorizados individual e coletivo.
Em estudos futuros, e para maior abrangência da aplicação, os resultados da análise de acessibilidade podem ser confrontados com mapas e dados socioeconômicos, como densidade e renda, complementando a caracterização do território quanto a sua vulnerabilidade. Assim, podem-se identificar áreas críticas para a mobilidade, ou seja, aquelas com maiores demandas e piores condições de atendimento.

\section{Referências}

Aguiar, F. O. (2010). Acessibilidade relativa dos espaços urbanos para pedestres com restrição de mobilidade (Tese de doutorado). Escola de Engenharia de São Carlos, Universidade de São Paulo, São Carlos.

Allen, W. B., Liu, D., \& Singer, S. (1993). Accessibility measures of U.S. metropolitan areas. Transportation Research Part B: Methodological, 27 (6), 439-449. http:// dx.doi.org/10.1016/0191-2615(93)90016-4.

Banister, D. (2008). The sustainable mobility paradigma. Transport Policy, 15(2), 73-80. http://dx.doi.org/10.1016/j. tranpol.2007.10.005.

Batista, E. D., Jr., \& Senne, E. L. F. (2000). TRANSIS: um novo método para avaliar o desempenho de sistemas de transporte urbano de passageiros. São Paulo: ANPET.

Bracarense, L. S. F. P., \& Ferreira, J. O. N. (2014). Índice proposto para comparação de acessibilidade dos modos de transporte privado e coletivo. In Anais do XVIII Congresso de Ensino e Pesquisa em Transportes. São Paulo: ANPET.

Brasil. Ministério das Cidades. Secretaria Nacional de Transporte e da Mobilidade Urbana. (2006). Brasil Acessível - (Programa Brasileiro de Acessibilidade Urbana. Caderno 2 - Construindo a Cidade Acessível, 167 p.). Brasília: SEMOB.

Brasil. (2012, 3 de janeiro). Lei ${ }^{\circ} 12.587$, de 3 de janeiro de 2012. Institui as diretrizes da Política Nacional de Mobilidade Urbana. Brasília: Diário Oficial União.

Bruton, M. J. (1979). Introdução ao planejamento dos transportes. Rio de Janeiro: Interciência; São Paulo: EDUSP.

Coriolano, G. P., Rodrigues, W., \& Oliveira, A. F. (2013). Estatuto da Cidade e seus instrumentos de combate às desigualdades socioterritoriais: o Plano Diretor Participativo de Palmas (TO). Revista Brasileira de Gestão Urbana URBE, 5(2), 131-145. http://dx.doi.org/10.7213/urbe.05.002.AC04. 
Costa, M. S. (2008). Um Índice de Mobilidade Urbana Sustentável (Tese de doutorado). Escola de Engenharia, Universidade de São Paulo, São Carlos. http://dx.doi. org/10.11606/T.18.2008.tde-01112008-200521.

Dalvi, M. Q. (1978). Behavioural modelling accessibility, mobility and need: concepts and measurement. In D. A. Hensher, \& P. R. Stopher (Eds.), Behavioural travel modelling (pp. 639-653). London: Croom Helm.

Davidson, K. B. (1995). Accessibility and isolation in transport network evaluation. In Proceedings of the VII World Conference on Transport Research (pp. 8-10). Sidney: The University of South Wales.

Deka, D. (2002). Transit availability and automobile ownership: some policy implications. Journal of Planning Education and Research, 21(3), 285-300. http://dx.doi. org/10.1177/0739456X0202100306.

Departamento Nacional de Infraestrutura de Transportes — DNIT. (2006). Manual de estudos de tráfego (384p.). Rio de Janeiro: DNIT.

Dias, R. F. (2008). Procedimento para elaboração do índice de acessibilidade com apoio de Sistema de Integração Geográfica - SIG (Dissertação de mestrado). Instituto Alberto Luiz Coimbra de Pós-graduação e Pesquisa em Engenharia, Universidade Federal do Rio de Janeiro, Rio de Janeiro.

Dodgson, J. S. (1974). Motorway investment, industrial transport costs, and sub-regional growth: a case study of the M62. Regional Studies, (8), 75-91. http://dx.doi. org/10.1080/09595237400185061.

Empresa Brasileira de Transportes Urbanos - EBTU. (1998). Planejamento da operação, diagnóstico do sistema existente: módulo de Treinamento, STPP Gerência do Sistema de Transporte Público de Passageiros (Vol. 2). Brasília: EBTU.

Ferraz, A. C. P., \& Torres, I. G. E. (2004). Transporte Público Urbano (2. ed., 410 p.) São Carlos: Rima.

Ferreira, M. A. G., \& Sanches, S. P. (2001). Índice de Qualidade das Calçadas - IQC. Revista dos Transportes Públicos, 23(9), 47-60.

Goto, M. (2000). Uma análise de acessibilidade sob a ótica da equidade: o caso da região Metropolitana de Belém (Dissertação de mestrado). Escola de Engenharia de São Carlos, Universidade de São Paulo, São Carlos.

Guimarães Rodrigues, T. (2011). Desenvolvimento de um indicador de acessibilidade para a avaliação de projetos de transporte sobre a exclusão social: estudo de caso da linha de metro 6 em São Paulo. In Anais do XXV Congresso de Pesquisa e Ensino em Transportes (12 p.). São Paulo: ANPET.

Gutierrez, A. (2012). Direito à Mobilidade. Direitos e Mobilidade. In Seminário Internacional de Direito Administrativo Contemporâneo e os Desafios da Sustentabilidade: Mobilidade Urbana, na Faculdade de Direito Mackenzie. São Paulo: Cidade em Movimento. Recuperado em 20 de maio de 2017, de http://cidadeemmovimento.org/ direito-mobilidade-direitos-e-mobilidade/

Hanson, S. (1995). Getting there: urban transportation in context. In S. Hanson (Ed.), The geography of urban transportation (pp. 3-25). New York: The Guilford Press.

Henk, R. H., \& Hubbard, S. M. (1996). Developing an index of transit service availability. Transportation Research Record: Journal of the Transportation Research Board, 1521, 12-19. http://dx.doi.org/10.3141/1521-02.

Hull, A. (2008). Policy integration: what will it take to achieve more sustainable transport solutions in cities? Transport Policy, 15(2), 94-103. http://dx.doi.org/10.1016/j. tranpol.2007.10.004.

Ingram, D. R. (1971). The concept of accessibility: a search for an operational Form. Regional Studies, 5(2), 101-107. http://dx.doi.org/10.1080/09595237100185131.

Januário, M. R. (1995). Procedimento para determinação de índices de acessibilidade de transporte e tratamento cartográfico dos mesmos (Dissertação de mestrado). Instituto Militar de Engenharia, Rio de Janeiro.

Jones, S. R. (1981). Accessibility measures: a literature review (38 p., TRRL LR 967 Monograph). Crowthorne, Berkshire: Transport and Road Research Laboratory.

Koenig, J. G. (1980). Indicators of urban accessibility: theory and application. Transportation Research, 9(2), 145-172.

Lemos, D. S. C. P. S. (2008). Contribuição metodológica para análise da acessibilidade sustentável (Qualificação em Engenharia de Transporte). Instituto Alberto Luiz Coimbra de Pós-graduação e Pesquisa em Engenharia, Universidade Federal do Rio de Janeiro, Rio de Janeiro.

Litman, T. (2008). Evaluating accessibility for transportation planning (49 p.). Victoria, Canada: Victoria Transport Policy Institute. Recuperado em 19 de janeiro de 2014, de http://www.vtpi.org/access.pdf

Melo, F. B. (2005). Proposições de medidas favorecedoras à acessibilidade e mobilidade de pedestres em áreas 
urbanas. estudo de caso: o centro de Fortaleza (Dissertação de mestrado). Universidade Federal do Ceará, Fortaleza.

Morris, J. M., Dumble, P. L., \& Wigan, M. R. (1979). Accessibility indicators for transport planning. Transportation Research Part A, General, 13A(2), 91-109. http://dx.doi. org/10.1016/0191-2607(79)90012-8.

Pires, F. M. A. (2000). Uma contribuição metodológica para a avaliação dos padrões de acessibilidade em redes rodoviárias (Dissertação de mestrado). Instituto Alberto Luiz Coimbra de Pós-graduação e Pesquisa em Engenharia, Universidade Federal do Rio de Janeiro, Rio de Janeiro.

Raia, A. A., Jr. (2000). Acessibilidade e mobilidade na estimativa de um índice de potencial de viagens utilizando redes neurais artificiais e sistemas de informações geográficas (Tese de doutorado). Escola de Engenharia de São Carlos, Universidade de São Paulo, São Carlos.

Saisana, M., Tarantola, S., Schulze, N., Cherchye, L., Moesen, W., \& Puyenbroeck, T. V. (2005). Knowledge economy indicators: state-of-the-art report on composite indicators for the knowledge-based economy. Ispra, Italia: JRC, European Commission Joint Research Centre.

Sales, L. H., Fo. (1996). O uso de indicadores de acessibilidade na eficácia de redes estruturais de transporte urbano (Tese de doutorado). Universidade Federal do Rio de Janeiro, Rio de Janeiro.
Santos, B. J. R. (2005). A qualidade no serviço do transporte público urbano. Goiânia: Pontifícia Universidade Católica de Goiás. p. 1-12.

Schoon, J., McDonald, M., \& Lee, A. (1999). Accessibility indices: pilot study and potential use in strategic planning. Transportation Research Record: Journal of the Transportation Research Board, 1685(1), 29-38. http:// dx.doi.org/10.3141/1685-05.

Tagore, M. R., \& Sikdar, P. K. (1995). A new accessibility measure accounting mobility parameters. In Proceedings of the VII World Conference on Transport Research. Sydney, Australia: University of New South Wales.

Vasconcellos, E. A. (2002). Transporte urbano nos países em desenvolvimento: reflexões e propostas (282р.). São Paulo: Annablume.

Vickerman, R. W. (1974). Accessibility, attraction, and potential: a review of some concepts and their use in determining mobility. Environment Planning, 6, 675-691. Wang, F. (2003). Job proximity and accessibility for workers of various wage groups. Urban Geography, 24(3), 253-271. http://dx.doi.org/10.2747/0272-3638.24.3.253.

Recebido: Jan. 25, 2017

Aprovado: Nov. 21, 2017 